\title{
Etude de l'effet androgénique de l'extrait au méthanol de Basella alba L. (Basellaceae) sur la fonction de reproduction du rat mâle
}

\author{
Edouard AKONO NANTIA ${ }^{1}$, Paul F. MOUNDIPA ${ }^{1}$, Nathalie Sara E. BEBOY ${ }^{1}$, \\ Thomas K. MONSEES ${ }^{2}$, Serge CARREAU ${ }^{3}$
}
${ }^{1}$ Laboratoire de Pharmacologie et de Toxicologie, Département de Biochimie, Université de Yaoundé I.
${ }^{2}$ Centre de Dermatologie et d'Andrologie, Université de Justus Liebig, Giessen, RFA
3 Laboratoire de Biochimie EA 2608-USC INRA 2006, IBFA, Université de Caen, France.

\section{RESUME}

Basella alba est une plante utilisée par les tradipraticiens de la province de l'Ouest Cameroun pour lutter contre l'asthénie sexuelle et l'infertilité chez l'homme. Les travaux antérieurs in vitro ont prouvé l'activité androgénique de son extrait au méthanol. Le présent travail porte sur la confirmation in vivo de son effet androgénique chez le rat mâle adulte. Cet extrait est quotidiennement administré (par gavage) pendant $\mathbf{3 0}$ jours aux doses de $0,0,1,1,10$ et $100 \mathrm{mg} / \mathrm{kg}$ respectivement à 5 groupes de 6 rats mâles.

Les animaux sont pesés tous les deux jours et au terme de 30 jours de traitement sont sacrifiés. Le sang est recueilli ; le foie et les organes de reproduction (testicules, épididymes, vésicules séminales, prostate) prélevẻs et pesés. Le sang est utilisé pour le dosage de la testostérone du sérum par 125 I-RIA et de quelques marqueurs de toxicité du foie et des reins. D'autres indicateurs de l'intégrité cellulaire sont également estimés dans le foie.

Les résultats montrent que l'extrait au méthanol de $B$. alba n'a pas d'effet sur le poids corporel des animaux ainsi que sur celui des organes de reproduction. Après 30 jours de traitement, l'effet de l'extrait au méthanol de $B$. alba sur la production de testostérone est significativement élevé $(p<0,0005)$ à la dose $1 \mathrm{mg} / \mathrm{kg}$, alors qu'à partir de $10 \mathrm{mg} / \mathrm{kg}$ d'extrait, ce niveau de testostérone baisse, mais reste élevé par rapport au témoin. Toujours au niveau du sérum, le taux de créatinine et l'activité de l'alanine aminotransférase ne montrent pas de variation significative entre les animaux témoins et ceux traités aux différentes doses d'extrait.
Cette observation est identique quant aux paramètres mésurés dans le foie : aniline hydroxylase, taux de protéines, de glutathion, d'aldéhyde malonique.

Ces résultats suggèrent que l'extrait au méthanol de $B$. alba a une activité androgénique in vivo qui est maximale à la dose $1 \mathrm{mg} / \mathrm{kg}$ de poids corporel.

Mots clés : Basella alba, activité androgénique, infertilité mâle, rat, testostérone, toxicité

Correspondance :

Pr. Paul F. MOUNDIPA - Laboratoire de Pharmacologie et Toxicologie, Université de Yaoundé I, B.P. 812, Yaoundé, Cameroun - $\mathrm{Tel}+2372016043 / 7310383$ -

Email pmoundipa@hotmail.com 


\section{INTRODUCTION}

L'infertilité est l'un des problèmes majeurs de santé qui affecte la vie socioculturelle de nombreux individus. Elle se définit comme la difficulté pour un couple d'avoir un enfant après une année de vie commune ponctuée de rapports sexuels fréquents et non protégés. Elle touche environ 8 à $14 \%$ des couples de part le monde, soit 50 à 80 millions d'individus. En Afrique subsaharienne où l'identité culturelle accorde une place de choix à la procréation, plus de $1 / 3$ des couples sont confrontés à ce problème au cours de leur existence $[3,6,16]$.

Les facteurs responsables de l'infertilité dans un couple sont difficiles à établir avec précision et impliquent souvent les deux sexes. Lejeune [8] a montré que l'homme et la femme se partagent la responsabilité de l'infertilité d'un couple à part sensiblement égale. La conséquence de la contribution du facteur masculin dans l'infertilité a été l'impulsion des recherches sur la santé reproductive mâle.

Le traitement de l'infertilité masculine par les plantes naturelles constitue de nos jours un mode de traitement de plus en plus sollicité [12]. Basella alba est une plante utilisée en médecine traditionnelle par les tradipraticiens de la province de l'Ouest Cameroun pour accroitre la virilité et la fertilité chez l'homme. Les études antérieures in vitro sur les morceaux de testicules et sur les cellules de Leydig de rats et de taureaux ont établi l'activité androgénique de l'extrait au méthanol de $B$. alba [10, 11]. Du fait des disparités entre l'effet pharmacologique d'un produit in vitro et celui in vivo, notre étude a essentiellement consisté à la vérification chez les animaux (rats mâles wistar albinos) de l'effet androgénique observé in vitro.

\section{MATERIEL ET MÉTHODES}

\section{Matériel végétal}

Les feuilles fraîches de $B$. alba (identifiée à l'Herbier National camerounais comme spécimen $N^{\circ} 40720$ ) ont été récoltées à Dschang (Ouest-Cameroun) au mois d'août 2005 et séchées à température ambiante puis finement broyées. L'extrait au méthanol est préparé par épuisement successif de la poudre obtenue à l'hexane, au chlorure de méthylène et au méthanol. Le rendement d'extraction au méthanol est de 3\%. L'extrait au méthanol est suspendu dans l'empois d'amidon de maïs $2 \%$ à une concentration de $10 \mathrm{mg} / \mathrm{ml}$ (extrait mère) et administré aux animaux aux différentes doses de $0 ; 0,1 ; 1 ; 10$ et $100 \mathrm{mg} / \mathrm{kg}$ de poids corporel.

\section{Protocole expérimental}

Les animaux utilisés sont les rats mâles wistar albinos adultes de 150 à $200 \mathrm{~g}$. Ces animaux sont élevés dans des cages en plastique et reçoivent de la nourriture (Laboratoire Nationale Vétérinaire, Garoua, Cameroun) et de l'eau du robinet ad libitum.

Les animaux répartis en 5 groupes de 6 rats chacun sont quotidiennement traités par gavage pendant 1 mois à l'extrait au méthanol de $B$. alba aux doses respectives de $0 ; 0,1 ; 1 ; 10$ et $100 \mathrm{mg} / \mathrm{kg}$ de poids corporel ; la dose $0 \mathrm{mg} / \mathrm{kg}$ correspondant aux animaux du groupe témoin traités avec le solvant de dilution de l'extrait (empois d'amidon $2 \%$ ). Le poids corporel des animaux est relevé tous les deux jours.

Après 30 jours de traitement, les animaux sont sacrifiés par décapitation et le sang récupéré. Le foie, les testicules, les épididymes, les vésicules séminales et la prostate sont prélevés et pesés.

Le sang est utilisé pour la préparation du sérum, lequel est conservé en aliquote au congélateur (à $\left.-40^{\circ} \mathrm{C}\right)$ en vue du dosage de la testostérone, de l'alanine aminotransférase (ALAT) et de la créatinine. Le foie est utilisé pour la préparation du surnageant postmitochondrial à $4^{\circ} \mathrm{C}$ par centrifugation à $9000 \times$ après broyage dans du tampon tris- $\mathrm{HCl} 50 \mathrm{mM}$ contenant du $\mathrm{KCl} 150 \mathrm{mM}$. Ce surnageant sera utilisé pour l'estimation du taux de protéines, de glutathion, d'aldéhydes maloniques et de l'activité de l'aniline hydroxylase.

\section{Tests biochimiques}

Le taux de testostérone sérique est déterminé par la méthode radio-immunologique utilisant le Kit Active Testostérone RIA (Testosterone [125] CT Kit, Lot : TK0609 LA, MP Biomedicals Germany). L'activité de l'alanine aminotransférase sérique est mesurée selon la méthode colorimétrique de Reitman et Frankel [13]. Le taux de créatinine sérique est déterminé par la réaction à l'acide picrique [2]. Le taux de protéines hépatiques est déterminé par la méthode de Gornall et al. [7] en utilisant l'albumine du sérum bovin comme étalon. Les taux de glutathion et d'aldéhydes maloniques hépatiques sont respectivement mesurés selon les méthodes de Ellman [5] et de Wilbur et al. [15].

L'activité de l'aniline hydroxylase dans le foie est évaluée selon la méthode décrite par Matsubara et Tochino [9].

\section{Analyses statistiques}

Les résultats sont analysés par le test de la variance (ANOVA) à un facteur et les moyennes des valeurs obtenues de différents groupes d'animaux sont comparées par le test de Student Newman Keuls. Les analyses sont faites à l'aide du logiciel statistique SPSS 10.0 . 


\section{RÉSULTATS}

1. Evolution du poids corporel des animaux au cours de l'expérimentation

La Figure 1 représente l'évolution du poids corporel des animaux durant 30 jours d'expérimentation. II en ressort que le poids corporel des animaux croît avec le temps. Aucune différence significative au seuil de probabilité $5 \%$ n'est cependant observée entre l'évolution pondérale des animaux du groupe témoin et celle des groupes tests.

\section{Poids du foie et des organes de reproduction en fonction du traitement}

La Figure 2 illustre le poids du foie, des testicules et de ses organes annexes des différents groupes d'animaux. Le traitement des animaux par l'extrait au méthanol de $B$. alba n'affecte pas le poids des organes, bien qu'une légère augmentation non significative soit observée sur le poids des épididymes, vésicules séminales et prostate des animaux traités par rapport aux témoins.

\section{Taux de testostérone sérique}

Le taux de testostérone sérique des différents groupes d'animaux est représenté à la Figure 3 . Après 30 jours d'expérimentation, la production de la testostérone croît avec la dose jusqu'à $1 \mathrm{mg} / \mathrm{kg}$ et décroît à partir de 10 $\mathrm{mg} / \mathrm{kg}$. Cette observation est illustrée par des pourcentages d'augmentation de $85 \%, 539 \%, 132 \%$ et $0 \%$ par rapport au groupe témoin respectivement pour des doses d'extrait au méthanol de $B$. alba de $0 ; 1 ; 1$ ; 10 et $100 \mathrm{mg} / \mathrm{kg}$. Par ailleurs, cette augmentation de testostérone sérique est hautement significative $(p<0,0005)$ chez les animaux recevant $1 \mathrm{mg} / \mathrm{kg}$ d'extrait de $B$. alba.

\section{Les paramètres biochimiques de toxicité}

Le Tableau 1 regroupe l'activité de l'alanine aminotransférase, de l'aniline hydroxylase, et les taux de créatinine, de protéines, d'aldéhydes maloniques et de glutathion dans les différents groupes d'animaux. D'après ces résultats, aucun des paramètres évalués ne varie significativement en fonction de la dose de l'extrait au méthanol de $B$. alba.

\section{DISCUSSION}

La testostérone est l'une des hormones essentielles de la physiologie masculine. En effet, elle est responsable du développement de la fonction testiculaire, de la stimulation et du maintien de la spermatogenèse, du développement de l'appareil musculaire, etc. [14]. Le rapport entre l'évolution de la testostérone et l'appareil musculaire, voire le poids corporel des animaux est bien établi. Mais au cours de notre étude, l'extrait au méthanol de $B$. alba n'a pas

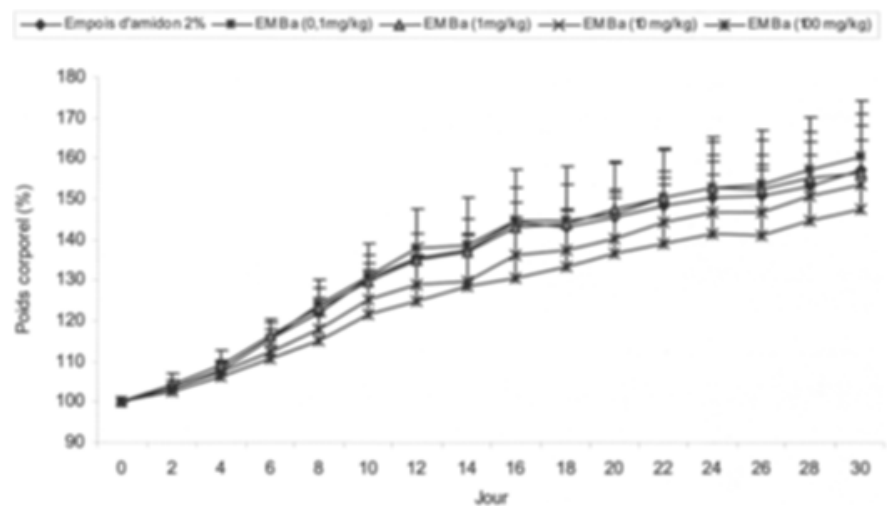

Figure 1: Evolution du poids corporel des animaux au cours de l'expérimentation. Chaque valeur représente la moyenne \pm écart type de 6 animaux par groupe. Les animaux ont été traités à l'extrait au méthanol de $B$. alba (EMBa) aux doses respectives de $0,0,1,1,10$ et $100 \mathrm{mg} / \mathrm{kg}$ de poids corporel.

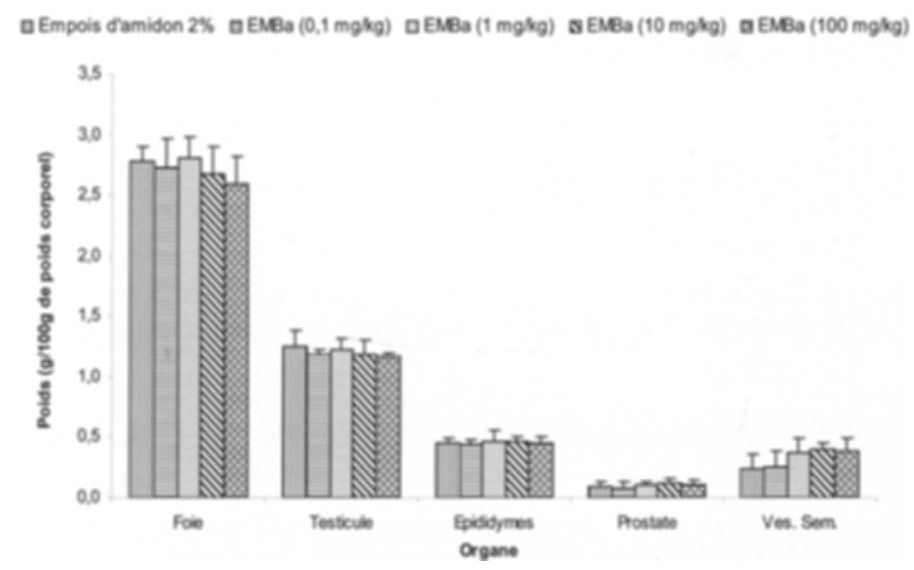

Figure 2 : Poids relatif des organes en fonction du traitement. Chaque valeur représente la moyenne \pm écart type de 6 animaux par groupe. Les animaux ont été traités à l'extrait au méthanol de $B$. alba (EMBa) aux doses respectives de 0 (témoin empois d'amidon 2\%), 0,1, 1, 10 et $100 \mathrm{mg} / \mathrm{kg}$ de poids corporel pendant 30 jours. (Ves Sem : Vésicules séminales).

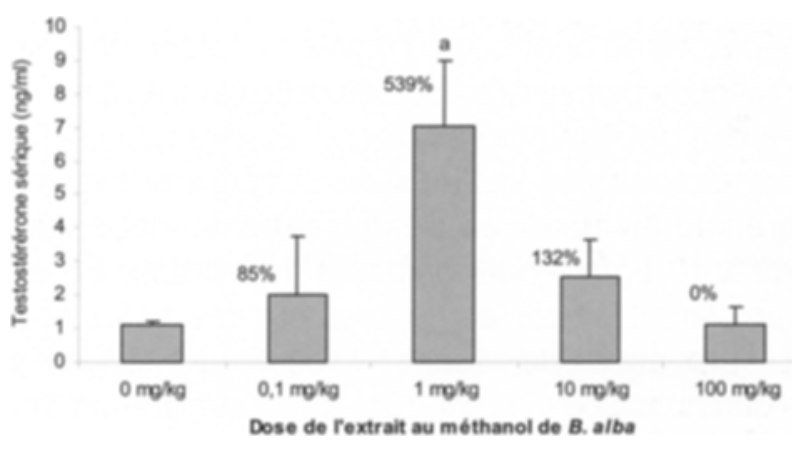

Figure 3: Taux de testostérone sérique en fonction du traitement. Chaque valeur représente la moyenne \pm écart type de 6 animaux par groupe. Les animaux ont été traités à l'extrait au méthanol de $B$. alba aux doses respectives de $0,0,1,1,10$ et $100 \mathrm{mg} / \mathrm{kg}$ de poids corporel. Les valeurs sont statistiquement différentes de celles du groupe témoin (recevant $0 \mathrm{mg} / \mathrm{kg}$ d'extrait). a:p<0,0005 (test ' $t$ ' de Student Newman Keuls). 
Tableau 1 : Activité de l'alanine aminotransférase (ALAT), de l'aniline hydroxylase et taux de créatinine, de protéines, d'aldéhydes maloniques et de glutathion chez les différents groupes de rats.

\begin{tabular}{ccccccc}
\hline $\begin{array}{c}\text { Extrait au } \\
\text { méthanol } \\
\text { de } B \text {. alba }\end{array}$ & $\begin{array}{c}\text { ALAT } \\
(\mathrm{UI} / \mathrm{L})\end{array}$ & $\begin{array}{c}\text { Créatinine } \\
(\mathrm{m} / \mathrm{l})\end{array}$ & $\begin{array}{c}\text { Protéines } \\
(\mathrm{mg} / \mathrm{g} \text { de foie })\end{array}$ & $\begin{array}{c}\text { Aniline } \\
\text { hydroxylase } \\
\left(10^{-3} \Delta \mathrm{DO} / \mathrm{h} / \mathrm{mg}\right. \\
\text { de protéine })\end{array}$ & $\begin{array}{c}\text { MDA } \\
\text { (nanomole/mg } \\
\text { de protéine) }\end{array}$ & $\begin{array}{c}\text { Glutathion } \\
\text { (mmole/mg de } \\
\text { protéine) }\end{array}$ \\
\hline $0 \mathrm{mg} / \mathrm{kg}$ & $41,94 \pm 10,9$ & $9,840,94$ & $183,37 \pm 34,15$ & $3,75 \pm 1,20$ & $0,044 \pm 0,015$ & $0,013 \pm 0,001$ \\
\hline $0,1 \mathrm{mg} / \mathrm{kg}$ & $39,80 \pm 6,02$ & $9,69 \pm 0,99$ & $174,24 \pm 73,58$ & $2,57 \pm 0,70$ & $0,049 \pm 0,022$ & $0,012 \pm 0,002$ \\
\hline $1 \mathrm{mg} / \mathrm{kg}$ & $41,22 \pm 5,50$ & $10,63 \pm 0,97$ & $180,87 \pm 8,65$ & $2,46 \pm 0,70$ & $0,042 \pm 0,025$ & $0,014 \pm 0,001$ \\
\hline $10 \mathrm{mg} / \mathrm{kg}$ & $38,32 \pm 3,40$ & $10,16 \pm 1,10$ & $173,42 \pm 26,37$ & $1,86 \pm 0,36$ & $0,023 \pm 0,008$ & $0,014 \pm 0,001$ \\
\hline $100 \mathrm{mg} / \mathrm{kg}$ & $48,91 \pm 12,6$ & $10,63 \pm 1,41$ & $214,60 \pm 22,27$ & $2,48 \pm 0,53$ & $0,025 \pm 0,014$ & $0,019 \pm 0,003$ \\
\hline
\end{tabular}

Chaque valeur représente la moyenne \pm écart type de 6 animaux par groupe. Les animaux ont été traités à l'extrait au méthanol de $B$. alba aux doses respectives de $0,0,1,1,10$ et $100 \mathrm{mg} / \mathrm{kg}$ de poids corporel. ALAT: alanine aminotransférase ; MDA : aldéhydes maloniques.

d'effet sur le poids corporel et des organes de reproduction.

La stimulation de la production de la testostérone de façon dose-dépendante jusqu'à $1 \mathrm{mg} / \mathrm{kg}$ d'extrait vient confirmer les observations in vitro de l'activité androgénique de l'extrait au méthanol de $B$. alba sur les coupes testiculaires et les cellules de Leydig de rat [10, 11]. Ce résultat pourrait s'appuyer sur la présence des composés de nature terpénique dans cet extrait [10] qui augmenteraient le pool en cholestérol, métabolite précurseur de la synthèse des androgènes.

La baisse de la testostéronémie aux doses d'extrait supérieures ou égales à $10 \mathrm{mg} / \mathrm{kg}$ témoigne sûrement d'un effet de désensibilisation de cette plante. Moundipa et al. [10] ont également observé in vitro que l'activité androgénique des cellules de Leydig traitées avec l'extrait au méthanol de $B$. alba est dose-dépendante jusqu'à $10 \mu \mathrm{g} / \mathrm{ml}$, puis baisse à $100 \mu \mathrm{g} / \mathrm{ml}$. En effet l'exposition des cellules de Leydig à des fortes doses de LH provoque une désensibilisation qui se traduit par une diminution de la production de la testostérone mais aussi de prégnénolone due soit à une des enzymes du clivage de la chaîne latérale du cholestérol et/ou soit à un défaut de couplage récepteur-cyclase membranaire $[1,4]$. Le mode d'action de l'extrait au méthanol de B. alba sur la stéroïdogenèse pourrait ainsi se rapprocher de celui de la LH.

Par ailleurs, il faudrait également considérer le facteur durée de traitement car les pourcentages d'augmentation de la testostérone sérique de $36 \%, 76 \%, 120 \%$ et $75 \%$ ont été obtenus au $15 \mathrm{e}$ jour du traitement des rats mâles avec des doses d'extrait de 0,1,1,10 et $100 \mathrm{mg} / \mathrm{kg}$ (données non publiées). Ainsi la dose $10 \mathrm{mg} / \mathrm{kg}$ pourrait être indiquée pour des traitements de courte durée, tandis que $1 \mathrm{mg} / \mathrm{kg}$ conviendrait pour des traitements de durée plus longue (supérieures ou égales à un mois).

L'évolution de l'effet pharmacologique d'extrait de plante ou d'une drogue donnée doit être soutenue par celle de son effet toxicologique. L'activité de l'alanine aminotransférase non significative entre les différents groupes d'animaux montre que l'extrait au méthanol de $B$. alba n'induirait pas de dommages sur la fonction hépatique, observation corroborée par l'activité de l'aniline hydroxylase et les taux d'aldéhydes maloniques et de glutathion cellulaire. Cet extrait serait également dépourvu de tout effet nocif sur la fonction rénale au regard du taux de créatinine sérique qui ne diffère pas entre les groupes.

\section{CONCLUSION}

L'extrait au méthanol de $B$. alba stimule la production de la testostérone chez les rats mâles wistar albinos de façon maximale à la dose de $1 \mathrm{mg} / \mathrm{kg}$. Cette dose serait donc intéressante pour des études ultérieures de l'activité androgénique de cette plante sur des patients humains souffrants de déficit androgénique ou d'hypoandrogénisme lié à l'âge. 


\section{Remerciements :}

Ce travail a été réalisé grâce au financement de la Bourse SALF 2006, La Fondation Internationale Pour la Science (F/2286-3F), et la DFG 445 KAM et GK 533.

\section{REFERENCES}

1. AQUILANO D.R., TSAI-MORRIS C.H., HATTORI M.A., DUFAU M.L. : Mitochondrial cholesterol availability during gonadotropin-induced Leydig cell desensitization. Endocrinol.,1985, $116:$ 1745-1754.

2. BARTELS H., BOHMER M., HEIERLI C. : Serum creatinine determination without protein precipitation. Clin. Chem. Acta. 1972, $37:$ 193-197.

3. BHATTI L.I., FIKREE F.F., KHAN A. : The quest of infertile women in squatter settlements of Karachi, Pakistan: a quantitative study. Soc. Sci. Med., 1999, $49: 637-649$.

4. COOKE J.L., WEST A.P. : The structure activity relationships of the luteinising hormone receptor. In : Nieschlag E, Habenicht $U$, eds. Spermatogenesis-fertilization-contraception. Shering Fondation worshop 4. Berlin, Springer Verlag, 1992, 21-31.

5. ELLMAN G. L. : Tissue sulfhydryl groups. Arch. Biochem. Biophys., 1959, $82: 70-77$

6. EMSLIE C., GRIMSHAW J., TEMPLETON A. : Do clinical guidelines improve general practice management and referral of infertile couples ? Br. Med. J., 1993, 306 : 1728-1731.

7. GORNALL A.G., BARDWILL G.S., DAVID M.M. Determination of serum proteins by means of Biuret reactions. J. Biol. Chem., 1949, $177:$ 751-766.

8. LEJEUNE H. : Panorama de l'infertilité masculine. Andrologie, 1999, $9: 9-27$.

9. MATSUBARA T., TOCHINO Y. : Inhibitory action of cyanide and aniline hydroxylase system. Febs Lett., 1975, $52: 77-80$.

10. MOUNDIPA F.P., BEBOY E.S.N., ZELEFACK F. et al. : Effects of Basella alba and Hibiscus macranthus extracts on testosterone production by adult rat and bull Leydig cells. Asian J. Androl., 2005, $7:$ 411-417.

11. MOUNDIPA F.P., SILVĖRE N., KAMTCHOUING P., TSAMO E., TCHOUANGUEP M.F., CARREAU S. : Effects of extracts from Hibiscus macranthus and Basella alba mixture on testosterone production in vitro in adult rat testes slices. Asian J. Androl., 2006, 8 : 111-114.

12. OMS : Stratégie de l'OMS pour la médecine traditionnelle pour 2002 - 2005. Organisation Mondiale de la Santé, Genève, 2002.

13. REITMANN S., FRANKEL S. : A colorimetric method for the determination of serum oxaloacetic pyruvic transaminases. Am. J. Clin. Path., 1957, 28 : 56-63.

14. WEINBAUER, G.F. NIESCHLAG E. : Testicular physiology of primates. In : Weinbauer G.F., Korte R. eds. Reproduction in non-human primates. Muenster, Waxmann, 1999 : 13-26.

15. WILBUR K.M., BERNHEIN F., SHAPIRO O.W. : The thiobarbituric acid reagent as a test for the oxidation of unsaturated fatty acid by various agents. Arch. Biochem. Biophys., 1949, 24 : 305-313.

16. WORLD HEALTH ORGANIZATION : Infertility : A tabulation of available data on prevalence of primary and secondary infertility. Geneva, WHO programme on maternal and family planning, division of family health, 1991.
Manuscrit reçu: janvier 2007 ; accepté février 2007

Bourse SAL 2006.

\section{ABSTRACT}

Androgenic activity of the methanol extract of Basella alba L. (Basellaceae) on male rat reproductive function

Edouard AKONO NANTIA, Paul F. MOUNDIPA, Nathalie Sara E. BEBOY, Thomas K. MONSEES,

\section{Serge CARREAU}

Basella alba is a plant used by some herbalists of the West province of Cameroon to enhance male virility and fertility. Previous in vitro studies have shown the androgenic activity of its methanol extract. This study therefore focused on confirmation of the androgenic activity of the methanol extract of $B$. alba in vivo in male rats. A suspension of this extract in corn starch was administered daily to 5 groups of rats at doses of $0,0.1,1,10$ and $100 \mathrm{mg} / \mathrm{kg}$ respectively for 30 days. The animals were weighed every second day during this period.

On the 31 st day of the experiment, the animals were sacrificed and blood was collected for serum assays. The liver and reproductive organs (testes, epididymides, seminal vesicles and prostates) were also collected and weighed. Serum testosterone level was determined by radio-immunoassay (125I-RIA) techniques, and various markers of liver and kidney toxicity (alanine aminotransferase and creatinine) were also assayed. Liver post-mitochondrial supernatant was used to estimate other markers of cell integrity, such as malondialdehyde, glutathione, and aniline hydroxylase activity.

The results showed that the methanol extract of $B$. alba has no effect on the weight of the animal and reproductive organs compared to the control. After 30 days of treatment, serum testosterone levels were significantly increased $(p<0.0005$ ) up to the dose of $1 \mathrm{mg} / \mathrm{kg}$, but decreased at higher dosages of the extract, although remained higher than that of controls. Aniline hydroxylase and aminotransferase activities, malondialdehyde, protein, glutathione and creatinine levels were not significantly altered by administration of the methanol extract of $B$. alba.

These results suggest that the methanol extract of $B$. alba has an in vivo androgenic activity with a maximum effect at the dose of $1 \mathrm{mg} / \mathrm{kg}$ body weight.

Key words: Basella alba, androgenic activity, male infertility, rat, testosterone, toxicity 